\title{
Defying the paradigm — rescue thrombolysis in a postoperative patient with pulmonary embolism
}

\author{
All India Institute of Medical Sciences, Jodhpur, India
}

Kunal Deokar, Ram Niwas, Nishant Chauhan, Gopal Chawla

Parenteral anticoagulation is recommended for patients of pulmonary embolism with intermediate to high early mortality risk. Rescue reperfusion is considered if signs of hemodynamic decompensation appear. Recent surgery within last 3 weeks is considered to be an absolute contraindication to thrombolytic therapy [1]. Percutaneous catheter-directed thrombolysis and surgical embolectomy can be done in such patients. However, they are not readily available. We hereby report a case of rescue thrombolysis in a post lower segment caesarean section (LSCS) patient with pulmonary thromboembolism. We successfully achieved thrombolysis in our patient with improvement in clinical and hemodynamic parameters and with no major bleeding from any site.

A 21-year-old married female was admitted to the intensive care unit with complaints of sudden shortness of breath and dizziness since last 6 hours. She had 2 episodes of syncope followed by spontaneous regaining of consciousness within 10 minutes. The patient had undergone LSCS 2 weeks back and had reduced mobility since then. On admission, she had tachypnea, tachycardia, was normotensive and hypoxic on room air. There were no signs of deep venous thrombosis. Arterial blood gases on ambient air revealed respiratory alkalosis with hypoxemia. Chest radiograph did not disclose any significant abnormality. Electrocardiogram showed right axis deviation and RV strain pattern. Her Wells and Revised Geneva scores were suggestive of intermediate risk of pulmonary embolism and as D-dimer was raised, urgent CT pulmonary angiography was performed which revealed a thrombus in both right and left pulmonary arteries and infarction involving the right middle lobe and posterior basal segment of the left lower lobe (Figure 1). 2D- Echocardiogram revealed dilatation of the right atrium and ventricle, positive McConnels sign with raised pulmonary artery systolic pressure of $60 \mathrm{~mm} \mathrm{Hg}$. NT-ProBNP was elevated (4054 pg/mL). Compression venous ultrasonography of bilateral lower limbs showed deep vein thrombosis of the right saphenous-femoral vein and popliteal vein. Her simplified pulmonary embolism severity index (sPESI) was 2 . As she had intermediate high early mortality risk, she was started on initial parenteral anticoagulation with unfractionated heparin. However, after few hours, she developed hypotension which was nonresponsive
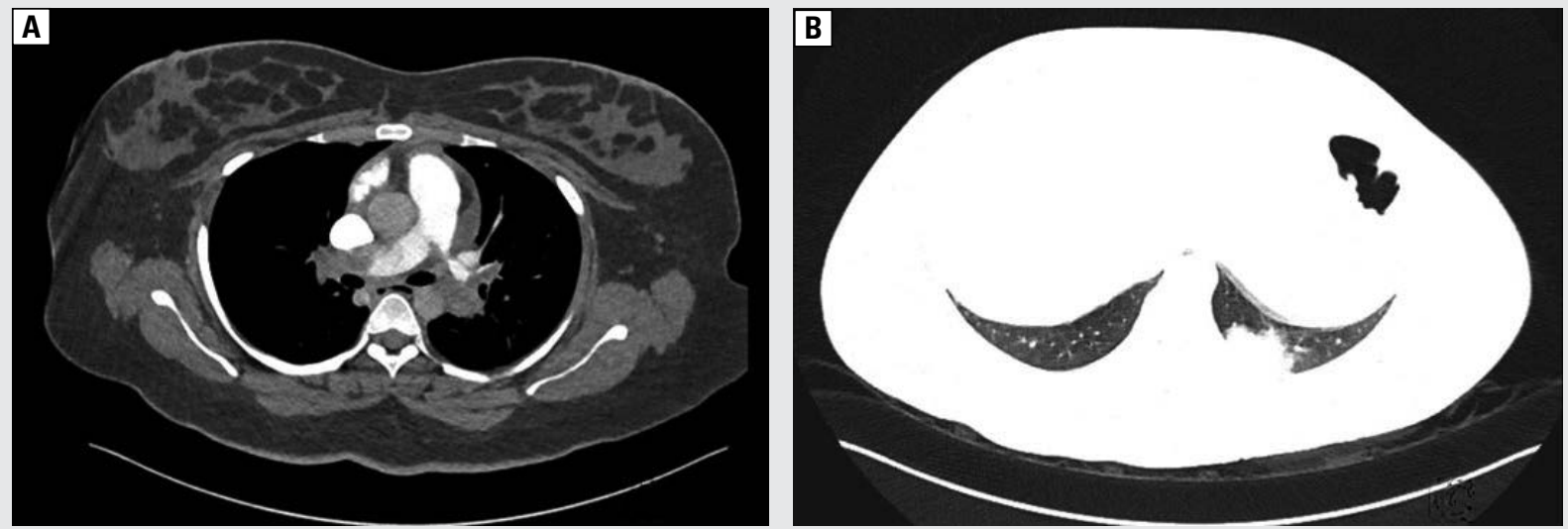

Figure 1. A. CTPA image showing a thrombus in both right and left pulmonary arteries; B. Lung window showing wedge-shaped infarct in the left lower lobe

Address for correspondence: Ram Niwas, All India Institute of Medical Sciences, Jodhpur, India; e-mail: miwasaiims@gmail.com

DOI: 10.5603/ARM.2020.0079

Received: 21.11.2019

Copyright (C) 2020 PTChP

ISSN 2451-4934

Conflict of interest: none declared 
Table 1. Case reports and studies of successful thrombolysis in patients of pulmonary embolism in the presence of contraindications

\begin{tabular}{|c|c|c|c|c|c|}
\hline & Author & Type of study & Year & Reason of contraindication & Agent used \\
\hline 1. & Cable DG et al. [2] & Case report & 2003 & Abdominal aortic aneurysm repair & Alteplase \\
\hline 2. & Koroneos A et al. [3] & Case report & 2007 & Intracerebral haemorrhage & Alteplase \\
\hline 3. & Han S et al. [4] & Case report & 2006 & Intracranial tumour & Alteplase \\
\hline 4. & Allport et al. [5] & Case report & 2008 & Stroke & Alteplase \\
\hline 5. & Zhang et al. [6] & $\begin{array}{l}\text { Retrospective study } \\
\qquad(\mathrm{n}=17)\end{array}$ & 2013 & Postoperative status & Urokinase \\
\hline 6. & Bottinor et al. [7] & Case report & 2014 & Haemorrhagic CVA & Alteplase \\
\hline
\end{tabular}

CVA - cerebrovascular accident

to crystalloid bolus infusion and sPESI deteriorated to 3. Repeated 2D-echo revealed bowing of the interventricular septum to the left in addition to previous findings.

Recent surgery within 3 weeks is considered as an absolute contraindication to systemic thrombolysis. A retrospective study and several case reports of patients with pulmonary embolism with various contraindications have shown that successful thrombolysis is possible in these patients [2-5] (Table 1).

In view of high mortality risk, and as catheter-directed thrombolysis was not immediately available, a decision to initiate rescue thrombolysis with streptokinase was taken. Risks and benefits of thrombolysis were discussed with the patient and her family members. After obtaining written informed consent, the woman was thrombolysed with streptokinase. After 24 hours, tachycardia and tachypnea resolved, she became normotensive and was weaned off oxygen. Repeated 2D-Echo revealed normal right heart chamber dimensions and SPAP reduced to $30 \mathrm{~mm} \mathrm{Hg}$. The patient did not develop any vaginal bleeding. Ultrasound of pelvis did not disclose any intrauterine or pelvic collection.

Our report highlights the fact that in cases of high-risk pulmonary embolism, an absolute contraindication to thrombolysis might become a relative contraindication. Systemic thrombolysis can be lifesaving in such patients.

\section{References:}

1. Konstantinides SV, Torbicki A, Agnelli G. 2014 ESC Guidelines on the diagnosis and management of acute pulmonary embolism: The Task Force for the Diagnosis and Management of Acute Pulmonary Embolism of the European Society of Cardiology (ESC). Eur Heart J. 2014; 35(43): 3033-3080, doi: 10.1093/eurheartj/ehu283.

2. Cable DG, Cherry KJ. Systemic thrombolytic therapy after recent abdominal aortic aneurysm repair: an absolute contraindication? Mayo Clin Proc. 2003; 78(1): 99-102, doi: 10.4065/78.1.99, indexed in Pubmed: 12528884.

3. Koroneos A, Koutsoukou A, Zervakis D, et al. Successful resuscitation with thrombolysis of a patient suffering fulminant pulmonary embolism after recent intracerebral haemorrhage. Resuscitation. 2007; 72(1): 154-157, doi: 10.1016/j.resuscitation.2006.06.019, indexed in Pubmed: 17084012.

4. Han S, Chaya C, Hoo GW. Thrombolytic therapy for massive pulmonary embolism in a patient with a known intracranial tumor. J Intensive Care Med. 2006; 21(4): 240-245, doi: 10.1177/0885066606287047, indexed in Pubmed: 16855059.

5. Allport LE, Butcher KS. Thrombolysis for concomitant acute stroke and pulmonary embolism. J Clin Neurosci. 2008; 15(8): 917-920, doi: 10.1016/j.jocn.2007.03.026, indexed in Pubmed: 18474426.

6. Zhang K, Zeng X, Zhu C, et al. Successful thrombolysis in postoperative patients with acute massive pulmonary embolism. Heart Lung Circ. 2013; 22(2): 100-103, doi: 10.1016/j.hlc.2012.08.055, indexed in Pubmed: 23068907.

7. Bottinor W, Turlington J, Raza S, et al. Life-saving systemic thrombolysis in a patient with massive pulmonary embolism and a recent hemorrhagic cerebrovascular accident. Tex Heart Inst J. 2014; 41(2): 174-176, doi: 10.14503/THIJ-12-3010, indexed in Pubmed: 24808778. 